\title{
Nano-materials and Nano-manufacturing
}

\author{
Nuo Yang ${ }^{1^{*}}$
}

DOI: $10.30919 / \mathrm{esmm} 5 \mathrm{f} 206$

This issue has nine interesting papers dedicated to nano-materials and nano-structured materials, including $0 \mathrm{D}$ quantum dots, $1 \mathrm{D}$ nanotubes, 2D graphene, thin films, nano-porous structures, nano-composites, and interfaces. Nano-materials and nano-structured materials have been widely studied for their brand new properties which are different from properties of their bulk counterparts, due to large surface-area-to-volume ratio, size effects, quantum confinements and so on.

Nanotubes are typical 1D materials. Song et al. (DOI: 10.30919/esmm5f193) explored and demonstrated that the multi-walled carbon nanotubes (MWCNTs) could serve as a promising adsorbent for methyl blue removal in wastewater. The MWCNTs-polyethylenimine adsorbent contributes to an enhancement of the adsorption capacity in methyl blue removal, where the maximum adsorption capacity is as high as $418 \mathrm{mg} / \mathrm{g}$ at $35{ }^{\circ} \mathrm{C}$. As a review, Liu et al. (DOI: 10.30919/esmm5f199) go through the carbon nanotube and boron nitride nanotube in structure, property and synthesis methods.

For 2D material, Tang et al. (DOI: 10.30919/esmm5f203) simulated the thermal conductivity of recently synthesized 2D materials, namely graphene embedded with periodic four-membered and eightmembered rings (GFERs). Both the length and the temperature dependence of GFERs' thermal conductivity are found to be different from that of the pristine graphene. An obvious thermal rectification in graphene-GFERs heterostructures is also found.

The abundant findings on nano-materials benefit from the progress of nano-manufacturing. Vikram et al. (DOI: 10.30919/esmm5f205) synthesized lead sulfide $(\mathrm{PbS})$ quantum dots $(\sim 6 \mathrm{~nm})$ using a costeffective and facile chemical synthesis method, namely ionic reaction. The strong size quantization was observed from cyclic voltammetry and absorption spectroscopy. Borate et al. (DOI: 10.30919/esmm5f202) investigated the effect of working gas pressure on the adhesive, structural, optical, morphology and electrical properties of Mo thin films to achieve better sputtering conditions. Furthermore, the performance of the fabricated Mo thin film was studied by using it as a working electrode in electrochemical deposition of $\mathrm{Cu}_{2} \mathrm{ZnSnS}_{4}$ thin films.

Besides nano-materials, there are also works on manufacturing nano-structured materials. Bai et al. (DOI: 10.30919/esmm5f195) demonstrated that the crystallization of the Polylactide (PLA) matrix could significantly affect thermal conductivity of the PLA/boron nitride (BN) nano-composites, comparing to amorphous PLA matrix. When $\mathrm{BN}$ content is $40 \mathrm{wt} \%$, the in-plane and through-plane thermal conductivities of the crystalline PLA/BN are $4.7 \mathrm{~W} / \mathrm{m} \cdot \mathrm{K}$ and 0.8

\footnotetext{
School of Energy and Power Engineering, Huazhong University of Science and Technology, 1037 Luoyu Ave., Wuhan, P. R. China 430074

*E-mail: nuo@hust.edu.cn
}

$\mathrm{W} / \mathrm{m} \cdot \mathrm{K}$, respectively. These are increased by $\sim 50 \%$ from those of amorphous PLA matrix, due to the increased phonon mean free paths. Iwe et al. (DOI: 10.30919/esmm5f197) fabricated nano-porous silicon structures with antireflection coating at extremely low technology cost, which can comfortably replace the conventional cells. The surface reflection in visible, infrared and ultraviolet regions has been drastically reduced to smaller than $0.1 \%$ by using gradient porous silicon technique. Zhang et al. (DOI: 10.30919/esmm5f201) synthesized a series of Sr-doped hierarchical porous nanofiber structure, such as $\mathrm{Li}_{1.2} \mathrm{Mn}_{0.54} \mathrm{Ni}_{0.13} \mathrm{Co}_{0.13-\mathrm{x}} \mathrm{Sr}_{\mathrm{x}} \mathrm{O}_{2}$. As cathode materials in a Li-ion battery, such materials can enhance the cyclic performance, suppress the voltage decay and stabilize the structure. Shanmugam et al. (DOI: 10.30919/esmm5f207) adopted two different electrochemical surface treatments, anodization and micro-arc oxidation (MAO). On a Ti alloy, these treatments may improve bonding strength of the interface between the metal and composite.

In summary, the works in this issue have studied a wide range of properties of nano-materials, such as adsorption capacity, thermal conductivity, photo-absorption capability, discharge capacity in batteries, etc. Besides, it also includes some works of advanced nanomanufacturing on $\mathrm{PbS}$ quantum dots, Mo thin films, PLA/BN nanocomposites, nano-porous $\mathrm{Si}$, pore structure $\mathrm{Li}_{1.2} \mathrm{Mn}_{0.54} \mathrm{Ni}_{0.13} \mathrm{Co}_{0.13-\mathrm{x}} \mathrm{Sr}_{\mathrm{x}} \mathrm{O}_{2}$ nanofibers, interfaces between $\mathrm{Ti}$ and a composite. These works can enhance the current understanding and lead to new research directions in nano-materials and nano-manufacturing. We are looking forward to many more exciting papers in coming issues.

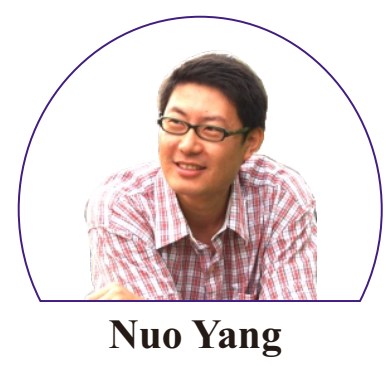

Nuo Yang is Chutian Distinguished Professor in School of Energy and Power Engineering, Huazhong University of Science and Technology. His research includes theories, simulations and measurements in micro/nanoscale, and his interests mainly focus on, but not limited to, phonon engineering, energy carriers' transport and energy conversions, such as thermal transport, thermal modulation, thermal device, thermal interfacial resistance, thermoelectrics and solar water evaporation/

condensation etc 